\title{
Perspectives of using Illumina MiSeq for identifying obligate symbionts of plants - arbuscular mycorrhiza fungi
}

\author{
Kryukov A.A. ${ }^{*}$, Gorbunova A.O. ${ }^{1,2}$, Machs E.M. ${ }^{3}$, Mikhailova Y.V. ${ }^{3}$, Rodionov A.V. ${ }^{3}$, \\ Yurkov A.P. ${ }^{1,2}$ \\ ${ }^{1}$ All-Russian Research Institute for Agricultural Microbiology, St. Petersburg, Russia \\ ${ }^{2}$ St. Petersburg State University, St. Petersburg, Russia \\ ${ }^{3}$ Komarov Botanical Institute, RAS, St. Petersburg, Russia \\ *e-mail:rainniar@rambler.ru
}

Arbuscular mycorrhiza fungi (AM) form one of the most common symbiosis with majority of land plants. AM fungi supply the plant with various mineral elements, primarily phosphorus, and improve the water supply. Search of the most symbiotic effective AM strains and the creation of microbial preparations on their basis is an important task for modern biology. The identification of AM is very difficult. This is primarily due to the high genetic AM polymorphism, as well as the difficulties of their cultivation without a host plant. The morphological identification of AM is often unreliable due to high number of cryptic species among AM. In recent years increases the number of AM biodiversity studies performed by modern NGS-based methods, in particular Illumina MiSeq. Using the Illumina MiSeq eliminates the need for a sequencing of large number of clones. Currently, there are still many questions in the identification of AM fungi. The most important of them are the choice of a genetic marker for the barcoding of AM fungi - conservative or variable sequences, as well as the choice of primers - specific for AM or universal. Another significant problem for molecular genetic identification of AM is DNA isolation. In our work, we successfully use universal primers ITS3 and ITS4 for the sequencing in Illumina MiSeq the 5.8SrRNA-ITS2 region, which contains both a conservative and variable regions. The effectiveness of identification of AM isolated from the roots of a host plant varies around 50 percent. When DNA is isolated from a spore, efficiency droped to 10 percent.

Acknowledgements: This work was supported by RFBR grant No. 18-016-00220 and RFBR grant No. 19-29-05275. 